\title{
III.
}

\section{Über Knorpelheilung nach aseptischen Verletzungen am hyalinen, von Perichondrium iiberzogenen, fertigen Knorpel.}

(Aus dem Pathologisch-anatomischen Institut der Königlichen Universität zu Padua.)

Von

Dr. Ramberto Malatesta.

(Hierzu Tafel V.)

Seit der Mitte des abgelaufenen Jahrhunderts sind oft Versuche angestellt worden, ob ein von den übrigen Geweben so verschieden gebautes Gebilde, wie der Knorpel, überhaupt und in welchem Maße fähig wäre, erlittene Substanzverluste wiederherzustellen. Trotz ausgedehnter Beobachtungen haben sich bisher noch keine übereinstimmenden Resultate ergeben, es bestehen nicht nur unter den älteren Autoren Gegensätze, oft. finden wir auch in den neuesten Arbeiten widersprechende Urteile.

Bei Untersuchungen über die Regenerationsfähigkeit des Knorpels müssen von vornherein die verschiedenen Arten des Knorpelgewebes auseinandergehalten werden, es muß also das mit Perichondrium versehene von dem ohne Perichondrium getrennt werden; unter ersterem sind der hyaline, der elastische und der Faserknorpel zu unterscheiden.

Man kann sich unschwer vorstellen, daß die feineren Vorgänge bei Regeneration des Gelenkknorpels anders sein werden als die der Rippen- und Kehlkopfknorpel, da bei diesen eine perichondrale Wucherung den Prozeß beeinflussen kann. Beide Arten sind nicht nur in älteren Arbeiten durcheinandergeworfen worden, auch in einigen ganz neuen ist eine Scheidung unterlassen, z. B. bei Matsuoka' ); er beschränkt in seiner Arbeit unter dem Titel „Die Regeneration des Knorpelgewebes" seine Experimente auf den elastischen Knorpel des Kaninchenohres.

1) Matsuoka, Die Regeneration des Knorpelgewebes. Dieses Archiv, Bd. 175 . 


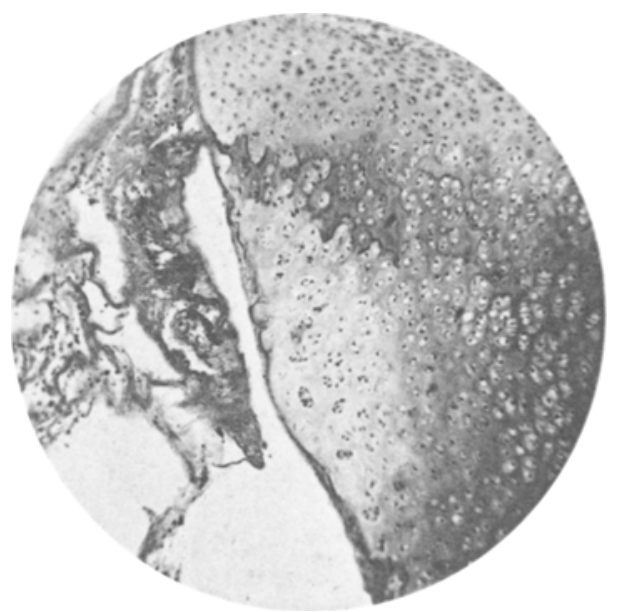

Fig. 1.

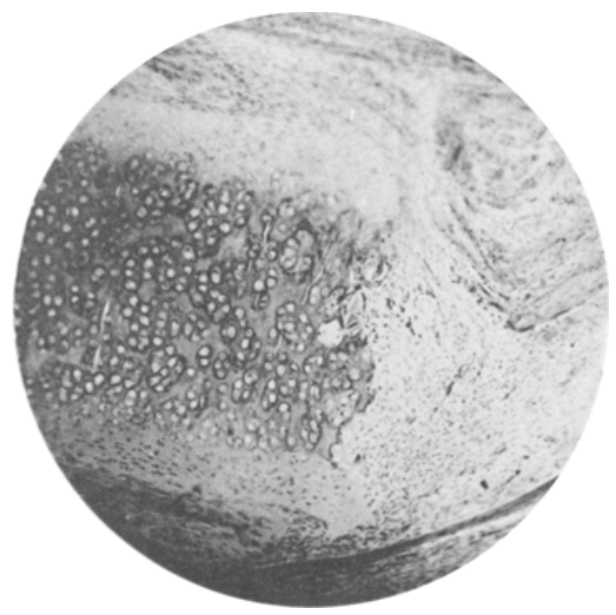

Fig. 3.

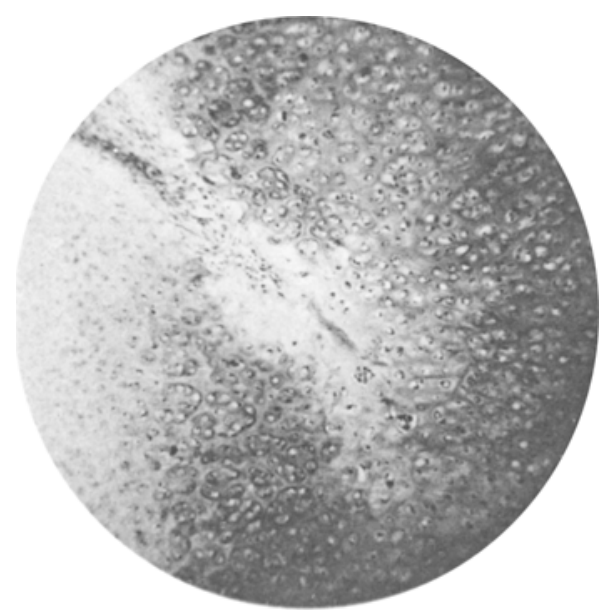

lig. 2.

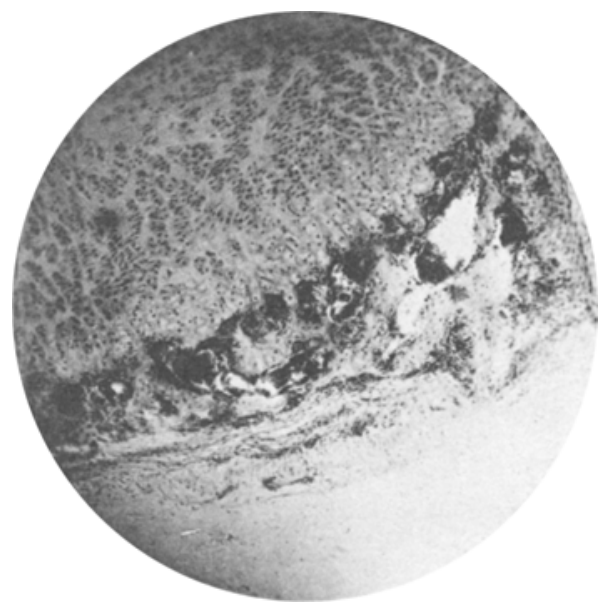

Fig. 4. 
Auf Grund dieser wenig geeigneten Objekte kommt er zu dem Schluß, daß der Knorpel keine Neigung zur Regeneration hat, daß bei allen Verletzungen ein Ersatz des Gewebes vom Perichondrium ausgeht, dạß dieses die einzige Matrix für die Heilung des Knorpels wäre.

Ich lege groBen Wert auf die Unterscheidung der beiden Arten: Knorpel mit und Knorpel ohne Perichondrium, weil das Fehlen dieser Einteilung die hauptsächlichsten Verschiedenheiten in dem Urteil der Autoren veranlaßt hat. Ein weiterer Umstand, dem Rechnung getragen werden muß, ist das Alter der Tiere; man muß annehmen, daß ebenso wie bei den übrigen Geweben auch beim Knorpel und beim Perichondrium die Regenerationsfähigkeit mit zunehmendem Alter sich ändert.

Ich halte es für überflüssig, die vielen über dieses Thema handelnden Arbeiten hier einzeln aufzuführen, man findet eine gute Literaturzusammenstellung in den neuesten Arbeiten bei Sieveking ${ }^{1}$ ), Matsuoka, Burci und Anzilotti ${ }^{2}$ ), Pennisi ${ }^{3}$ ), Seggel $\left.{ }^{4}\right)$, Fasoli ${ }^{5}$ ).

Ich beschränke mich darauf, die Punkte, über welche heute noch Meinungsverschiedenheiten existieren, hier anzuführen. Die eine Frage ist die, ob der verletzte Knorpel, unabhängig vom Perichondrium, einer aktiven Proliferation fähig sei oder ob er sich, wie die meisten annehmen, passiv verhalte. Eine weitere Frage: ob der Callus, der sich an der Stelle der Verletzung bildet, bindegewebig oder fibrocartilaginär sei oder aus hyalinem Knorpel bestände. Die am meisten diskutierte Frage ist endlich die, ob die Heilung vermittelst Knochengewebes zustande kommen könnte.

1) Sieveking, Beiträge zur Kenntnis des Wachstums und der Regeneration des Knorpels. Schwalbes morpholog. Arbeiten. 1893.

2) Burci u. Anzilotti, Contributo sperimentale allo studio delle lesioni traumatiche delle cartilagini e dei relativi processi per la riparazione. Memorie chirurgiche in onore di Enrico Bottini. 1902.

3) Pennisi, Über den ProzeB der Wundheilung des Knorpels. Policlinico, sez. chirurg. 1904.

4) Seggel, Studien über Knorpelwunden und--defekte. D. Zeitschr. $f$. Chirurgie, Bd. 75, 1904.

5) Fasoli, Ober das Verhalten des Knorpels bei Verletzungen. Archiv. per le scienze mediche, Bd. 29, 1905. 
Meine Untersuchungen stellte ich an Rippenknorpeln 8 bis 10 Monate alter Kaninchen an. Das Tier wurde in Rückenlage fixiert, die Haare am Thorax abgeschnitten, die Gegend sterilisiert, die Haut, die Pectoralis major und minor durchschnitten in der Richtung ihrer Muskelfasern, die Rippenknorpel bloßgelegt. Mit einem ganz feinen Bisturi schnitt ich den Rand oder die Vorderfläche eines Knorpels an, oder ich nahm ein keilförmiges Stück heraus, kratzte ein wenig Perichondrium ab oder führte einen Schnitt quer durch. Letztere Operation gab sehr häufig Veranlassung zur Verschiebung der beiden Enden; um sie genau gegenüberzustellen, nahm ich einige Male ganz feine Seide. Solche Operationen machte ich sowohl an den gröberen oberen als auch an den feineren an der unteren Apertur des Thorax seitlich gelegenen Knorpeln. Im allgemeinen nahm ich an einem Tiere mehrere Operationen vor, bisweilen auch in mehreren Zeiten. Ich vermied es immer, auf die Wunden antiseptische Substanzen zu bringen, um nicht die zugehörigen Gewebe zu reizen. Nur zwei der Fälle kamen zur Eiterung, über diese berichte ich nicht, alle anderen heilten ohne Anschwellung und ohne Drüsenschwellungen; die Hautwunde heilte schnell per primam. Bei der Sektion fand ich, abgesehen von den 2 schon erwähnten Fällen, niemals eine bestehende oder abgelaufene Eiterung. 20 Tiere habe ich in dieser Weise operiert, im ganzen 50 Wunden hergestellt, die zur mikroskopischen Untersuchung kamen. Die Tiere wurden nach $6,7,8,12,14,18,25,35,45,53,60,68,75,80,100$, 120 Tagen getötet.

Gleich darauf kamen die Knorpelstücke auf 48 Stunden in 10prozentiges Formalin, darauf wurden sie 24 Stunden lang in 5prozentiger Salpetersäure entkalkt, sodann in Celloidin eingebettet, das m. E. besser als Paraffin dafür geeignet ist. Mit Hämatoxylin-Eosin oder nach van Gieson wurde gefärbt.

Ich möchte vorwegnehmen, daß sich bei allen von mir operierten Tieren ebenso wie bei gleichaltrigen nicht operierten eine Verkalkung der Rippenknorpel vorfand. Diese Verkalkung verhielt sich verschieden, je nachdem die zarteren (8.-12.) oder die dickeren (1.-7.) Rippen untersucht wurden. Bei ersteren nahm die Verkalkung die ganze Knorpelsubstanz ein 
mit Ausnahme einer dünnen subperichondral gelegenen Schicht, während bei den dickeren Knorpeln noch eine zentrale Zone frei blieb. Um diese sah man mit bloßem Ange eine Art Manschette, welche bei Querschnitten an frischen Präparaten in Form eines Ringes erschien; bei Behandlung mit Salzsäure verschwand der Ring unter Bildung von Gasblasen. Pennisi hatte ähnliche Beobachtungen erhoben, er legte ihnen aber kein Gewicht bei. Was das Vorhandensein von Fetttropfen in den Knorpelzellen anbetrifft, was als physiologischer Ausdruck des fertigen und alten Knorpels betrachtet wurde, so muß ich bemerken, daß in den Teilen, die dem Verkalkungsprozeß entsprechen, die Tropfen beträchtlich größer waren, wie man an Osmiumpräparaten nachweisen konnte. Die Ablagerung der Kalksalze in der Grundsubstanz und die Vermehrung der Fetttropfen in den Zellen sind beides zweifellos Zeichen regressiver Metamorphose und zeigen, daß die Rippenknorpel erwachsener Kaninchen eine verschiedene Lebensfähigkeit haben: eine geringere in den feineren Rippen, eine höhere in den dickeren und zwar in ihrem Zentrum. Wir werden sehen, wie dies bei der Wundheilung in bezug auf aktive Proliferation von großer Bedeutung ist.

Eine ausführliche Beschreibung der vielen mikroskopischen Präparate würde langweilig sein und zu Wiederholungen Anlaß geben, ich will daher nur zusammenfassende Resultate bringen, besonders insoweit die schon erwähnten noch ungelösten Fragen in Betracht kommen.

An vollständigen wie an unvollständigen Schnittwunden durch die Knorpel der dickeren Rippen sieht man nach 6-8 Tagen an denselben Stücken progressive und regressive Prozesse. Bei vollständigem Durchtrennen zeigen die meist übereinandergeschobenen Enden eine Verdickung des Perichondriums und der subperichondralen Schicht, man kann dann leicht den stufenweisen Übergang des Perichondriums zum eigentlichen Knorpelgewebe erkennen. In diesem Zeitabschnitt hat das Perichondrium noch keine Tendenz, in der Längsrichtung der Rippe zu wuchern, die freien Enden der durchschnittenen Rippe bleiben ohne Bedeckung oder haben unregelmäBig fibrinöse Massen an der Schnittfläche. Die Verkalkungszone unter 
der subperichondralen Schicht zeigt bemerkenswerte Veränderungen in der Nähe der Verletzung und zwar ausschließlich regressiven Charakters: Atrophie und Schwund der Knorpelzellen. In noch wohlbegrenzten Knorpelkapseln sieht man keine Zellen mehr oder höchstens noch mit Hämatoxylin ganz schwach bläulich gefärbte homogene Massen, zweifellos Reste zerstörter Zellen. Die Kapseln an der Schnittfläche sind frei geblieben, so daß in der Verkalkungszone das resecierte Knorpelstück nicht rechtwinklig, sondern kreisförmig eingezogen erscheint. Die verkalkte Grundsubstanz zeigt keinerlei Veränderungen.

Deutlicher und interessanter sind die Veränderungen der zentralen nicht verkalkten Zone. Auf eine bestimmte Strecke hin hat sie fast vollständig ihre Färbbarkeit verloren, während sie weiterhin sich wie gewöhnlich ziemlich intensiv mit Hämatoxylin färben läßt. In der entfärbten Zona, die ich „Zona pallida" nennen möchte, sind gleichzeitig pro- und regressive Zustände zu sehen. Letztere bestehen darin, daß einzelne Kapseln leer oder mit Resten, die durch Zerstörung von Zellen entstanden sind, gefüllt sind, erstere dagegen haben Zellen mit gut gefärbtem Kern, umgeben von ziemlich reichlichem Protoplasma, in rundlichen oder ovalen Gruppen von 4-8-12 Stück angeordnet (Fig. 1, Taf. V). Da sie dicht nebeneinander liegen, haben diese Zellen dreieckige oder polygonale Formen, die äußeren haben eine kreisbogenförmige Begrenzung. Es handelt sich hier zweifellos um "isogene Gruppen" wachsender Knorpelzellen, die noch nicht von Kapseln umgeben sind.

Bei unvollständigen Schnittwunden erkennt man, ebenfalls nach 6-8 Tagen, daß die Wundhöhle, die in den Präparaten die Form eines $V$ hat, zu einem guten Teil von jungem Granulationsgewebe eingenommen wird, das von dem umgebenden lockeren Gewebe seinen Ursprung nimmt. An den Rändern besteht eine leichte Verdickung des Perichondriums und in der Verkalkungszone Zeichen der Degeneration, so wie es oben beschrieben ist. - Die lymphatischen Elemente dringen da, wo sie mit dem Rand der Verkalkungszone in Berührung treten, in die offenen Kapseln ein und können bei oberflächlicher Betrachtung irrtümlicherweise für „isogene" Gruppen von Knorpel- 
zellen gehalten werden. - Das Ende der Spalte, das in der Mitte der Rippe liegt, wird von einer mit Eosin färbbaren Substanz eingenommen, welche aller Wahrscheinlichkeit nach die Fibrinreste der bei dem Trauma entstandenen Blutung darstellt. Der Knorpel in der Umgebung ist ungefärbt, die Zellkapseln sind leer oder enthalten höchstens Reste von in Auflösung begriffenen Zellen. Nur ab und zu finden sich Haufen zelliger Elemente, die nichts anderes als ,isogene“ Gruppen analog den beschriebenen darstellen (Fig. 2, Taf. V).

Hiernach ergibt sich, daß sich die subperichondrale, die Verkalkungs- und die zentrale Zone verschieden verhalten. Die erste nimmt an Dicke zu, ohne ihre Art zn verändern, sie zeigt unter sich verschiedene Zellen, die sich einzeln ihrer Kapseln entledigen, die zweite geht nur regressive Prozesse ein, auf die wir später noch zurückkommen, die dritte zeigt dagegen gleichzeitig re- und progressive Prozesse.

Das blasse, fast ungefärbte Aussehen des verkalkten Knorpels hängt vọ einem Degenerationsprozeß desselben $a b$, bei starker Vergrößerung kann man an einzelnen Stellen direkt eine Auffaserung des Knorpels in Fibrillen erkennen, die sog. asbestartige Degeneration. Die Zellen werden dabei sicher atrophisch.

Alle Autoren haben in der Nachbarschaft der Wunden eine Degeneration des Knorpels gefunden, aber nur wenige erkennen eine aktive Proliferation seitens des präexistierenden Knorpels, unabhängig von den Zellen des Perichondriums, an. Von neveren Untersuchern leugnen Marchand und Pennisi vollständig eine solche Proliferation, Burci und Anzilotti lassen sie dagegen zu. Für mich gibt es keinen Zweifel, daß die Zellen wirklich gewacherte Knorpelzellen sind; sie finden sich in gewissem Abstande vom Rande der Wunde, sie haben keine Beziehung zu dem Granulationsgewebe in der Wundhöhle, wie man sich an Seriensehnitten überzeugen kann, deswegen sowie wegen ihrer Gestalt muß man es als ausgeschlossen ansehen, daß sie von dem kleinzelligen Granulationsgewebe, das an den beiden Stümpfen wuchert, herkommen.

Es sind vielmehr Neubildungen des unbeschädigt gebliebenen Knorpels, oder sollten sie sich in situ aus einer Wucherung 
von Zellen bilden, die einer im Zustande der Atrophie befindlichen Knorpelzone angehören? Sowenig wir in dem Teil des normalen Knorpels, der allmählich in die "Zona pallida" übergeht, „isogene“. Gruppen neugebildeter Zellen finden, und sowenig wir ein Recht haben, durch die Knorpelgrundsubstanz eine Zellenwanderung zuzulassen, so bleibt nur der Schluß übrig, daß der atrophische Knorpel an ihrer Bildung beteiligt ist. Daß dieser trotz vorgeschrittener Atrophie noch Elemente enthält, die neue Knorpelzellgruppen hervorsprießen lassen können, geht aus folgendem Versuche hervor: Ich durchschnitt bei einem Kaninchen den 7. Rippenknorpel vollständig und machte an einem der Stümpfe dicht neben und parallel zu dem vorigen Schnitt einen zweiten vollständigen, der bis zur subperichondralen Zone der anderen Seite reichte. Nach 12 Tagen untersuchte ich das Stück und fand, daß der Knorpel zwischen den beiden Schnitten den Charakter der Zona pallida hatte; fast alle Zellen waren verschwunden, die Kapseln leer, an einigen Stellen lagen jedoch Zellgruppen mit gut färbbarem Kern, die alle Zeichen neugebildeter ,isogener" Gruppen an sich trugen. Dieser Versuch beweist, daß dieselbe Knorpelzone, die sich in Degeneration befindet, zur selben Zeit Zeichen von Regeneration aufweisen kann. Eine weitere Frage ist nun die folgende: Können diese neugebildeten Zellen an die Stelle des atrophischen Knorpelgewebes treten und den durch die Wunde herbeigeführten Substanzverlust ersetzen? Aus der Untersuchung der nicht vollständig verkalkten Rippenknorpel ergibt sich, daß die „Zona pallida" sich allmählich umwandelt, die atrophischen Zellen verschwinden, die neugebildeten vermehren sich, trennen sich voneinander, die Grundsubstanz fängt an, rings um die einzelnen Elemente eine Hämatoxylinfärbung anzunehmen, so daß die Zellen wie von einem bläulichen Hof umgeben erscheinen. Dieser Hof vergrößert sich fortschreitend, die Zellen verteilen sich unter Zunahme der Grundsubstanz im Gewebe, und nach einer 60- bis 80tägigen Periode ist keine Spur der „Zona pallida" mehr vorhanden, sie hat sich in normalen Knorpel verwandelt.

Bei den vollständigen Wunden mit oder ohne Verschiebung der Stümpfe: machen die Regenerationserscheinungen an dem 
Rand der Wunde Halt und nehmen nicht an dem Konsolidierungsprozeß der Stümpfe teil, welcher, wie wir sehen werden, vermittelst eines vom Perichondrium und umgebenden Bindegewebe herstammenden Gewebes zustandekommt. An den unvollständigen Wunden der dickeren Rippenknorpel kann sich dagegen im Grunde der Wunde, einem ganz engen Spalt, der Ersatz aus der Knorpelwucherung selbst bilden. Wenn Fibrin darin liegt und dieses nicht von außen kommt und von kleinzelligen Wucherungen seinen Ursprung nimmt, so geht der Heilungsprozeß der Zona pallida auch auf die Fibrinmassen über, so daß die Wunde, die in den ersten Tagen nach der Operation die Form eines spitzen $V$ hatte, nach 60-80 Tagen die Gestalt eines abgestumpften Kegels angenommen hat; die Spitze ist dann schon durch normalen Knorpel gebildet, während der Rest der Höhle noch von Granuationsgewebe ausgefüllt wird.

Die dünnen Rippenknorpel des erwachsenen Kaninchens verhalten sich während des Heilvorgangs ganz anders. Sie sind, wie schon erwähnt, vollständig im Innern verkalkt, sie verhalten sich wie die Verkalkungsschichten der dickeren Knorpel. Es kommen daher keine progressiven Prozesse an ihnen vor, an jeder Wunde, mag sie vollständig: den Knorpel durchsetzen oder unvollständig sein, sieht man Atrophie und Schwund der Zellen in der Umgebung, die Kapseln, die an der Schnittfläche liegen, werden geöffnet, verlieren ihre Zellen, es nisten sich kleinzellige Elemente in ihnen ein, die vom Granulationsgewebe herkommen (Fig. 3, Taf. V). Die verkalkte Grundsubstanz, welche keine raschen Veränderungen eingeht, bricht in der Nähe des Wundrandes auseinander, in die Fissuren dringen Zellen infiltrierend vor, dringen in leere Kapseln ein und können dann das Bild einer "isogenen" Neubildung vortäuschen. Diese Infiltrationszellen bringen das atrophische Knorpelstück zur Sequestrierung, es treten polynucleäre Zellen auf, auch richtige Riesenzellen, welche kleine Stücke der Grundsubstanz in sich aufnehmen. Sie liegen ziemlich reichlich an den zackigen Wundrändern oder dringen auch in die Spalten der Grundsubstanz vor, morphologisch und fanktionell den Riesenzellen des Knochens analog. Verkalkte Knorpelstückchen finden 
sich auch oft eine Strecke von der Wunde entfernt, mitten in jungem Bindegewebe vor, welches das Narbengewebe der beim Sehnitt verletzten weichen Teile bildet. Auch nach 100 Tagen ist der atrophische Teil des verkalkten Knorpels noch nicht vollständig entfernt.

In neuster Zeit hat Mori ${ }^{1}$ ) am Ohrknorpel des Kaninchens Versuche angestellt und in den leeren Kapseln an der Schnittfläche Zellen gefunden, die er rom Granulationsgewebe der Umgebung der Wunde herleitet und nicht für Knorpelproliferationen hält; bei meinen Versuchen ist der Befund damit übereinstimmend, die Erklärung für die verkalkten Knorpelschichten ebenfalls, für die nicht verkalkten centralen Teile der dickeren Rippenknorpel aber, aus den angeführten Gründen, vollkommen entgegengesetzt.

Das abweichende Verhalten der obersten sieben Rippenknorpel beim erwachsenen Kaninchen gibt eine hinreichende Erklärung für die verschiedenen Resultate der Experimentatoren.

Alle Autoren sind darüber einig, daß bei der Heilung von Knorpelwunden das Perichondrium und das umgebende Bindegewebe die größte Bedeutung hat, Uneinigkeiten bestehen nur über die Art des Gewebes, das dadurch gebildet wird. Aus meinen Untersuchungen ergibt sich, daß nach einem vollständig den Rippenknorpel durchsetzenden Schnitt zuerst um diesen herum ein infolge der Blutung fibrinöses Material gefunden wird, welches alsbald durch eine Bindegewebswucherung ersetzt wird; sie nimmt ihren Ursprung von der äußeren Schicht des Perichondriums und von lockerem Gewebe in der Nähe, das bei der Operation entweder direkt verletzt oder einfach gereizt wird. Auch an den Intercostalmuskeln sind Proliferationsvorgänge zu finden, da wo sie bei der Operation zerschnitten wo rden waren, man findet die charakteristische Kernvermehrung am Sarkolemm.

Die Bindegewebswucherung, die man in den ersten Tagen findet, wechselt an Ausdehnung innerhalb ziemlich weiter Grenzen.

1) Mori, Studien über Knorpelregeneration nach experimentellen Untersuchungen am Kaninchenohr. Deutsche Zeit. f. Chirurgie, Bd. 76, Heft 2, 3, 1905. 
Bei vollständigen Schnitten mit Auseinanderstehen der Stümpfe sind diese von einer Masse neugebildeten Bindegewebes umgeben, dasselbe bildet gewissermaßen einen Callus. Nach 12-30 Tagen geht der Callus in der. Nachbarschaft des Perichondriums eine fibrocartilaginäre Umwandlung ein; während das Perichondrium eine Neubildung hyalinen Knorpels verursacht, der aus stammesgleichen Zellgruppen zusammengesetzt ist und in dichter Verbindung mit dem alten Knorpel steht, sieht man an der Außenseite desselben im Bindegewebscallus eine deutliche Färbbarkeit der zelligen Substanz mit Hämatoxylin; die Zellen stellen sich in Reihen auf, die zur Knorpelachse parallel liegen und umgeben sich mit einer Kapsel, die Intercellularsubstanz läßt bei starker Vergrößerung einen streifigen Bau erkennen. - Diese fibrocartilaginäre Umwandlung beginnt stets in der Nähe des Perichondriums und breitet sich von da weiter aus. Nach 40-50 Tagen können die von Rippenknorpel bedeckten, mit Bindegewebscallus vollkommen umgebenen Stümpfe durch ein dazwischenliegendes Stück Faserknorpel miteinander fest verbunden sein. Zunächst bleibt der Faserknorpel auf eine schmale Zone beschränkt, später breitet er sich von da auf den ganzen Bindegewebscallus aus, dieser kann für längere Zeit, vielleicht für immer bestehen bleiben, im allgemeinen wandelt er sich aber in hyalinen Knorpel um. Aber nicht immer braucht der sich bildende hyaline Knorpel den Faserknorpel als Vorstufe, er kann direkt aus dem Bindegewebe entstehen, und zwar beginnt dieser Prozeß gleichfalls in der Nähe des Perichondrinms. Wenn der Callus sehr groß ist, kann die Knorpelbildung unregelmäßig vor sich gehen, an einigen Stellen bildet sich dann vielleicht Faserknorpel, an anderen bleibt mehr oder weniger gefäßreiches Bindegewebe, an noch anderen endlich findet sich hyaliner Knorpel. Es ist nicht möglich, genau die Zeit anzugeben, innerhalb welcher sich der Bindgewebscallus in Knorpel verwandelt; auch nach 120 Tagen sah ich noch einen Teil des Callus bindegewebig. In einigen Fällen schließlich bleibt die Knorpelbildung ganz aus, der Callus liefert, wie alle Narbengewebe, faseriges Bindegewebe; an den untersten Rippenknorpeln war das häufiger. 
Bei unvollständig den Knorpel durchsetzenden Wunden verlaufen auch die regeneratorischen Prozesse viel milder. Das Fibrin in der Wunde wird in den meisten Fällen von jungem Bindegewebe durehsetzt, dieses hat aber weniger Neigung, sich in Knorpel zu verwandeln, als bei den vollständigen Wunden. Erst nach 50-60 Tagen habe ich an ihm eine Knorpelbildung. im Beginn wahrnehmen können und zwar außen an der Wunde, so daß man zu gewisser Zeit einen Zustand trifft, wo außen Knorpel, innen Bindegewebe die Wundhöhle einnimmt. Noch nach 120 Tagen fand ich in einem Fall die Wunde von fibrösem Gewebe erfüllt, das wahrscheinlich einer weiteren Umwandlung nicht mehr fähig war.

Bei der Bildung des hyalinen Knorpelgewebes scheint das Perichondrium einen direkten Einfluß zu haben und zwar sowohl bei der Bildung auf dem Wege über den Faserknorpel als auch bei direkter Entstehung aus dem Bindegewebscallus; die Knorpelbildung beginnt stets in der Nähe des Perichondriums. Man könnte deswegen glauben, daß es sich um eine wirkliche Knorpelwucherung handle, welche das Bindegewebe ersetzen sollte; die mikroskopische Untersuchung der Präparate spricht aber dagegen; man kann darin den allmählichen Übergang des Bindegewebes zum Knorpel verfolgen, was bei einem Ersatz des einen Gewebes durch das andere nicht möglich wäre. Übrigens ist die Fähigkeit des Bindegewebes, sich in Knorpel umzuwandeln, im allgemeinen anerkannt, in unserem Falle würde das benachbarte Perichondrium den besonderen Reiz abgegeben haben. Diese Wirkung des Perichondriums sollte von mir experimentell mit der Zerkratzung desselben bewiesen werden, ich fand aber an Stelle des zerstörten Perichondriums, auch nach langer Zeit, nur ein Granulationsgewebe, in dem sich keine Spur einer knorpeligen Umwandlung nachweisen lieB.

Gewöhnlich fand ich im Knorpel unter der Schnittwunde, ebenso bei teilweiser Zerkratzung desselben, daß zwischen der Schwere der Verletzung und dem Regenerationsvermögen ein umgekehrtes Verhältnis besteht. Die stärkste Knorpelhautwucherung, die sich in starker Verdickung der subperichondralen Knorpelzone ausdrückte, erhielt ich bei leichten Verletzungen, bei denen das Perichondrium gar nicht oder nur ganz wenig 
zerstört war; andererseits trat bei Wunden, die durch den Knorpel hindurch bis auf die andere Seite desselben subperichondral sich erstreckten, in dem Perichondriam der nicht durchschnittenen Seite eine Neubildung von hyalinem Knorpel auf, während auf der Seite der Wunde die Knorpelwucherung ziemlich unbedeutend war.

Eine letzte Modifikation, die der Callus eingehen kann, ist die Verknöcherung. Pathologisch-anatomische Untersuchungen über diesen Gegenstand rühren von Rokitansky, Ollier, Cornil und Ranvier, Nélaton und anderen her, sie betreffen don Callus bei Rippenknorpelbrüchen erwachsener und alter Menschen. Tierexperimente ergaben aber keine Ubereinstimmung damit, Pennisi fand eine Verknöcherung, Tizzoni ${ }^{1}$ ) Mare hand, Burci und Anzill otti und andere leugnen sie nach Versuchen an gebrochenen oder resecierten Rippenknorpeln.

Nach meinen Experimenten tritt die Verknöcherung ziemlich regelmäßig ein, nach 50-60 Tagen erkennt man sie, ausnahmsweise auch schon einmal nach 35 Tagen; immer ist der Callus schon vorher knorpelig geworden, die Verknöcherung tritt dann in derselben Weise ein, wie bei den langen Röhrenknochen an den Epiphysen (vgl. Fig. 4, Taf. V); die Knorpelsäulen stehen senkrecht zum äußeren Callusrand, die Ossifikationszone erscheint immer auf der Höhe dieses Randes, mit dem Fortschreiten des Prozesses allmählich weitergehend. Die Gefäßschlingen der Verknöckerungszone stammen aus dem umliegenden weichen Gewebe. Im Innern des Knorpelcallus sah ich niemals die Verknöcherung beginnen. Der neue Knochen ist spongiös, hat zarte Bälkchen, an denen man den lamellösen Bau gut erkennen kann, ebenso die verzweigten Knochenkörperchen.

Bei genauerer Untersuchung bemerkt man jedoch, daß der in Rede stehende Prozeß, analog dem bei der normalen enchondralen Ossifikation, beachtenswerte Anomalien darbietet. Es sind: 1. die wenig regelmäßige Anordnung der Knorpelsäulen,

1) Tizzoni, Utber die normale und pathologische Histologie der hyalinen Knorpel. Archiv. per le scienze mediche. Vol. $\mathrm{II}^{0} 1878$. 
welche eine zu breite Ausdehnung einnehmen und eine schlecht begrenzte Zone bilden; 2. unregelmäßige Verteilung der Verkalkungszone; 3. unregelmäßiges Vordringen der Gefäße, die sich meistens nicht in einer Fläche vorfinden; 4. die in dem neugebildeten Knochen verstreuten Knorpelinseln; 5 . unregelmäßige Verteilung der Osteoblasten an der Wand der neuen Markhöhle. Ganz dieselben Anomalien finden sich bei der Rachitis leichteren Grades ebenfalls vor als Zeichen eines gestörten und unvollkommenen Verknöcherungsprozesses.

Nicht alle Wunden an verschiedenen Knorpeln eines Tieres geben einen knöchernen Callus, so daß man nicht von individueller Disposition sprechen kann; es besteht vielmehr eine Beziehung mit der Art der Verletzung: wenn ein Maximum der perichondralen Reizung mit einem Minimum von Knorpelhautzerstörung einhergeht, wenn also die Knorpelwucherung größer zu werden versprach, dann besteht auch eine größere Wahrscheinlichkeit auf Verknöcherung des Callus. Man kann daher leicht verstehen, wie ein Rippenknorpelbruch zuerst einen knorpeligen und dann einen knöchernen Callus hervorrufen kann, letzterer nimmt die Form einer Manschette an, innerhalb welcher die beiden knorpeligen Stümpfe liegen, die an der Knochenbildung keinen Anteil mehr haben. Bei meinen Tieren war der Knochenersatz im Knorpel nur partiell, wahrscheinlich hätten aber längere Beobachtungszeiten auch stets Knochenbildung ergeben. Nach dem 53. Tage fand ich Verknöcherung in der Hälfte der operierten Rippenknorpel.

Fassen wir die Ergebnisse unserer Untersuchungen über die Heilung am hyalinen, ausgebildeten, mit Perichondrium bekleideten Knorpel zusammen, so lassen sich die folgenden Sätze aufstellen:

1. Der hyaline, mit Perichondrium versehene Knorpel ausgewachsener Tiere (Kaninchen) reagiert bei Verletzungen je nach seiner Lebensfähigkeit verschieden: ist er ganz verkalkt, so treten nur regressive Prozesse auf, welche zur Ausstoßung des die Wunde umgebenden Knorpelstückes führen; ist er nur teilweise verkalkt, so vollzieht sich in dem nicht verkalkten Teile zunächst eine Atrophie, später beginnen in derselben Zone einige Elemente zu wuchern, sie setzen sich ganz allmählich 
an die Stelle der atrophischen oder zugrunde gegangenen Zellen und bringen auf diese Weise eine Wiederherstellung des atrophischen Teils zustande.

2. Nur bei kleinen unvollständigen Schnittwunden am nicht vollständig verkalkten Knorpel kann eine teilweise Heilung. durch das Knorpelgewebe selbst zustande kommen; in allen andern Fällen nimmit der Knorpel selbst nicht am Verschluß des Loches oder an der Verbindung der beiden Stümpfe teil.

3. Ausnahmsweise produciert das am Heilungsprozesse teilnehmende, die Wunde umgebende Knorpel- und Bindegewebe hyalinen Knorpel bezw. junges Bindegewebe, welch letzteres später eine permanente fibröse Narbe bildet, häufiger jedoch allmählich in Faserknorpel und schließlich in hyalinen Knorpel umgewandelt wird.

4. Die Umwandlung des Bindegewebscallus in Knorpel vollzieht sich unter dem Einfluß des Perichondriums.

5. Die knorpelerzengende Eigenschaft des Perichondriums ist bei geringen Reizungen am stärksten, bei etwas weiter gehenden Zerstörungen fehlen Knorpelwucherungen gänzlich, ja es bleibt sogar jede Heilung aus.

6. Nach längerer Zeit kann der Knorpelcallus verknöchern, analog der physiologischen Verknöcherung, es bildet : sich spongiöser Knochen.

7. Der vorher vorhandene Knorpel hat an der Verknöcherung keinen Anteil.

Meinem verehrten Lehrer Professsor B on o me sage ich zum Schluß meinen ergebensten Dank für die Leitung meiner Versuche.

\section{Erklärung der Abbildungen auf Taf. V.}

Fig. 1. Vollständige Durchschneidung des 7. Rippenknorpels. 6. Tag: Die Abbildung zeigt den Rand des einen Stumpfes; man sieht daran von links nach rechts die Verdickung der subperichondralen Zone, die verkalkte Zone in regressivem Zustande und die Zona pallida, in welcher die stammesgleichen Gruppen neugebildeter Zellen dicht nebeneinander stehen.

Fig. 2. Unvollständiger Schnitt am 6. Rippenknorpel. 6. Tag: Die Abbildung gibt das Ende der Wundhöhle wieder, die in der Dicke des nicht verkalkten Knorpels gelegen ist. Um das Ende der 
mit Fibrinresten gefüllten Wunde liegt eine bloße Zone („Zona pallida"), in ihr stammesgleiche Gruppen neugebildeter Zellen.

Fig. 3. Vollständige Durchschneidung des 8. Rippenknorpels. 25. Tag; Am Rande des einen, auf dem Bilde sichtbaren Stumpfes bemerkt man Zeichen von Atrophie an den Zellen und eine Auflösung der verkalkten Grundsubstanz.

Fig. 4. Vollständige Durchschneidung des 5. Rippenknorpels. 68. Tag: Verknöcherung des knorpeligen Callus.

\title{
IV. \\ Über lokales und allgemeines Amyloid.
}

(Aus der Inneren Abteilung des Krankenhauses Bethanien zu Berlin.)

\author{
Von \\ Dr. Edens. \\ Assistenzarzt an der Abteilung.
}

(Hierzu Tafel VI.)

Das Verhältnis lokaler Amyloidbildung zur allgemeinen Amyloidentartung darf ein besonderes Interesse beanspruchen in solchen Fällen, die beide Prozesse nebeneinander aufweisen. Wichmanns ${ }^{1}$ Definition der lokalen Amyloiderkrankung bietet allerdings für diese Möglichkeit keinen Raum, da nach ihr der übrige Körper frei rom Amyloid sein muß. Ich habe schon an früherer Stelle gegen diese Auffassung geltend gemacht, daß sie einer sehr wohl möglichen Koinzidenz lokaler und allgemeiner Amyloiderkrankung nicht gerecht. werde. Als Stiutze dieses theoretischen Einwandes fand sich ein einziger, von Less er ${ }^{3}$ (nicht Hildebrandt ${ }^{4}$, wie durch ein Versehen in meiner ersten Arbeit angegeben ist) beschriebener Fall in der Literatur. Kürzlich nun hatte ich Gelegenheit, bei der Sektion eines Falles von sehr ausgedehnter und alter allgemeiner Amyloiderkrankung einen Tumor der sechsten linken Rippe zu finden, der sich bei der näheren Untersuchung als eine lokale Amyloidbildung charakterisierte. Die Begründung dieser Auffassung werde ich später geben. Da tumorförmiges Amyloid des Knochenmarks in der hier vorliegenden Form bisher noch nie beschrieben worden ist, so verdient der Fall zunächst schon als einziger 